\title{
Functionality versus strength - has functional selection taken place in the case of the ecdysteroid receptor response element?
}

\author{
Iwona Grad, Marian Kochman and Andrzej Ożyhar ${ }^{\bowtie}$ \\ Institute of Organic Chemistry, Biochemistry and Biotechnology, Division of Biochemistry, \\ Wroctaw University of Technology, Wroctaw, Poland
}

Received: 11 July, 2002; accepted: 28 August, 2002

Key words: 20-hydroxyecdysone, ecdysteroid receptor, ultraspiracle, nuclear receptor, Drosophila, protein-DNA interaction

\begin{abstract}
Nuclear receptors are ligand-dependent transcription factors responsible for controlling differentiation, growth and development of higher eukaryotes. Three amino acids within the recognition $\alpha$-helix of the DNA-binding domain of the nuclear receptors constitute the so-called "P-box" which determines response element specificity. In the ultraspiracle (Usp) protein, which together with EcR forms the heterodimeric ecdysone receptor, the P-box residues are E19, G20 and G23. Substitution of E19, the most characteristic amino acid for estrogen receptor-like P-boxes, with alanine showed that the mutation did not appreciably alter the affinity of the wild-type Usp DNA-binding domain (UspDBD ${ }_{\mathrm{WT}}$ ) for a probe containing natural ecdysone response element $\left(h s p 27_{w t}\right)$. Since in many cases $\mathrm{E} 19$ contacts a $\mathrm{G} / \mathrm{C}$ base pair in position -4, which is absent in $h s p 27_{w t}$, we analysed the interaction of UspDBD $\mathrm{WT}_{\mathrm{WT}} \mathrm{E} 19 \mathrm{~A}$ and other P-box region mutants with the $h s p 27_{w t}$ derivative which contains a G/C instead of an T/A base pair in position -4. UspDBD ${ }_{\mathrm{WT}}$ exhibited higher affinity for this element than for $h s p 27_{w t}$. Moreover, a different interaction pattern of P-box region mu-
\end{abstract}

\footnotetext{
$\boldsymbol{\Phi}_{\text {This work was supported by grants to A. Ożyhar from the State Committee for Scientific Research }}$ (KBN, Poland) grant 3 P04B 00923 and the Center for Biomonitoring, Biotechnology and Protection of Ecosystems of Lower Silesia.

${ }^{\circledR}$ Corresponding author: Andrzej Ożyhar, Institute of Organic Chemistry, Biochemistry and Biotechnology, Division of Biochemistry, Wrocław University of Technology, Wybrzeże Wyspiańskiego 27, 50-370 Wrocław, Poland; phone/fax: +71 320 6333; e-mail: ozyhar@kchf.ch.pwr.wroc.pl

Abbreviations: DBD, DNA-binding domain; 20E, 20-hydroxyecdysone; EcR, product of the EcR gene; EcRDBD, DNA-binding domain of the product of the $E c R$ gene; EcRE, 20-hydroxyecdysone response element; EMSA, electrophoretic mobility shift assay; GST, glutathione $S$-transferase; $h s p 27_{w t}, 20$-hydroxyecdysone response element consisting of an imperfect palindrome from the promoter region of the Drosophila hsp27 gene; $h s p 27_{-4 G}$, $h s p 27_{w t}$ with base pair G/C in position -4; RE, response element; RXR, retinoic acid receptor; Usp, product of the ultraspiracle gene; UspDBD, DNA binding domain of the product of the ultraspiracle gene.
} 
tants was also observed. Thus we conclude that the E19 residue of UspDBD is not involved in any $\mathrm{hsp} \mathrm{Z}_{w t}$ sequence-discerning contacts. However, substitution of the hsp27 ${ }_{w t} \mathrm{~T} / \mathrm{A}$ base pair in position -4 with $\mathrm{G} / \mathrm{C}$ generates target sequence with distinct functional characteristics and possibly with a new specificity. These results could serve as a basis for understanding the role of the presence of a T/A or G/C base-pair in the position -4 in the two types of ecdysone response elements found in nature.

20-Hydroxyecdysone (20E) is a steroid controlling larval moulting, metamorphosis and reproduction in insects and other Ecdysozoa (Kozlova \& Thummel, 2000; Sluder \& Maina, 2001). The hormone acts via its receptor - a heterodimer of two proteins (Yao et al., 1992) - the products of the $E c R$ (Koelle et al., 1991) and ultraspiracle (Oro et al., 1990) genes (EcR and Usp, respectively). EcR and Usp belong to the nuclear hormone receptor superfamily, which comprises ligand-dependent transcription factors with a characteristic domain structure (Evans, 1988). However, ecdysone receptor holds a unique position among other receptors. Although its ligand is a steroid, the functional ecdysone receptor is a heterodimer (Yao et al., 1992) unlike vertebrate steroid hormone receptors (Gehring, 1998). Moreover, it seems to bind its response elements only upon ligand binding (Thomas et al., 1993), which is not true of the receptors heterodimerising with the vertebrate retinoic acid receptor (RXR) (Torchia et al., 1998) - a Usp ortholog. The most conserved part of nuclear receptors is the DNA binding domain (DBD) with two characteristic zinc-binding modules (Freedman et al., 1988). Following the binding of the proper ligand, nuclear receptors interact with specific DNA sequences called response elements (REs). REs are derived from a common consensus sequence which during evolution was duplicated and modified, and now comprise two types of sequences - direct repeats, characteristic for heterodimeric receptors and palindromes, preferred elements for vertebrate steroid hormone receptors (Gronemeyer \& Laudet, 1995). The duplicated structure of the REs is in consistence with the nature of the nuclear receptors often forming homo- or heterodimers upon binding their REs. The main structure responsible for the specific recognition of the proper RE is the $\alpha$-helix of the first zinc-binding module of the DNA-binding domain (Renaud \& Moras, 2000). It has been demonstrated that only a few amino acids within the $\alpha$-helix are responsible for the recognition of the correct RE. Three of them, which constitute the so-called "P-box", are the most important ones for the mechanism of RE discrimination (Green et al., 1988; Mader et al., 1989; Nelson et al., 1999).

The proteins in the nuclear receptor superfamily can be divided into subgroups according to their P-box sequences. EcR and Usp belong to the estrogen receptor-like group with the P-box amino acids E19, G20 and G23 (Umesono \& Evans, 1989). Unlike vertebrate heterodimeric receptors, which prefer RE organized as direct repeats (Renaud \& Moras, 2000 ), the $20 \mathrm{E}$ receptor preferentially mediates transcription through highly-degenerated pseudopalindromes with a single intervening nucleotide (Riddihough \& Pelham, 1987; Antoniewski et al., 1993; Antoniewski et al., 1994; Antoniewski et al., 1995; Lehmann \& Korge, 1995; Lehmann et al., 1997). Our previous work (Niedziela-Majka et al., 2000) on EcR and Usp DBDs suggested polarity of the UspDBD/EcRDBD heterodimer on the natural pseudo-palindromic 20E RE (EcRE) from the Drosophila hsp27 gene (Riddihough \& Pelham, 1987; Ożyhar et al., 1991), with UspDBD acting as a specific anchor that preferentially binds the $5^{\prime}$ half-site of this element locating the heterocomplex in a defined orientation (see Fig. 1 for information on UspDBD and hsp27 EcRE sequences).

Here we demonstrate that mutation of E19, the most characteristic amino acid found in all receptors containing estrogen receptor-related P-box sequences (Umesono \& Evans, 
1989), did not appreciably alter the affinity of UspDBD and the UspDBD/EcRDBD heterocomplex for $h s p 27$ element. It was unexpected since E19 forms the most comprehensive, structurally conserved and specificity-determining contacts in other receptors (Schwabe et al., 1993; Rastinejad et al., 1995; Zhao et al., 1998; Meinke \& Sigler, 1999; Rastinejad et al., 2000; Zhao et al., 2000). In order to determine the basis for the insensitivity of UspDBD towards the mutation of its supposedly most critical P-box amino acid, we analyzed the interaction of wild type UspDBD (UspDBD ${ }_{\mathrm{WT}}$ ) and its E19A mutant with the hsp27 element and a derivative thereof containing a $\mathrm{G} / \mathrm{C}$ base pair in position -4 instead of T/A. Our results indicate that the E19 residue of UspDBD seems to be not involved in any hsp27 element sequence-discerning contacts. Interestingly, natural selection tends to favor EcREs which similarly as the hsp27 element contain a T/A base pair in the position -4 , although the substitution of the T/A base pair with G/C generates target sequence with higher affinity and distinct functional properties.

\section{MATERIALS AND METHODS}

Bacterial strain and plasmid vector. The plasmid pGEX-2T (Amersham, Biosciences, Germany) containing $l a c I^{q}$ gene was used for expression of DBDs as fusions with Schistostoma japonicum glutathione $S$-transferase (GST-DBD) (Smith \& Johnson, 1988). For GST-DBDs production Escherichia coli strain BL21(DE3)pLysS (Studier, 1991) (Novagen, U.S.A.) was used.

The construction of DBDs-expression vectors; site-directed mutagenesis. Construction of the expression plasmids for the wild type Drosophila melanogaster Usp GST-DBD (pGEX-UspDBDWT) was described previously (Niedziela-Majka et al., 1998). PCR-based megaprimer mutagenesis protocol (Barik, 1995) was applied to introduce alanine codons. The plasmid pGEX-UspDBD ${ }_{\mathrm{WT}}$ was used as a template and the sequences of the mutated DNA fragments were verified by dideoxy sequencing.

Overexpression and purification of the wild-type and mutant proteins. The expression of GST-DBDs and purification of GST-free wild type and mutated UspDBD, were performed as described previously (Niedziela-Majka et al., 1998; Grad et al., 2001). The designations of the respective mutant UspDBD, are based on the amino acid single letter code (e.g., E19A = Glu-19 $\rightarrow$ Ala).

DNA-binding assays. The sequences of the ds oligonucleotides used in the study (see Fig. 1B) are based on the sequence from the $D$. melanogaster hsp27 gene promoter (Riddihough \& Pelham, 1987; Ożyhar et al., 1991). Electrophoretic mobility-shift assays (EMSAs) (Fried \& Crothers, 1981) were performed under conditions previously elaborated (Grad et al., 2001).

Protein concentration. Concentration of the purified proteins was determined spectrophotometrically at $280 \mathrm{~nm}$ using absorption coefficients calculated according to Gill and von Hippel (1989).

\section{RESULTS AND DISCUSSION}

The E19 residue of nuclear receptor DBDs with estrogen receptor-related P-boxes forms most the defined, structurally conserved and specificity-determining contacts with the response elements. In many receptors E19 accepts a hydrogen bond from the $\mathrm{N} 4$ of the cytosine of base pair G-4/C-4 and in many cases it contacts the adenine of the T-3/A-3 base pair through a water molecule and also takes part in other complex interactions responsible for the response element recognition (Schwabe et al., 1993; Rastinejad et al., 1995; Zhao et al., 1998; Meinke \& Sigler, 1999; Rastinejad et al., 2000; Zhao et al., 2000) (see Table 1A). Surprisingly, the introduced mutation of E19 did not appreciably alter the affin- 


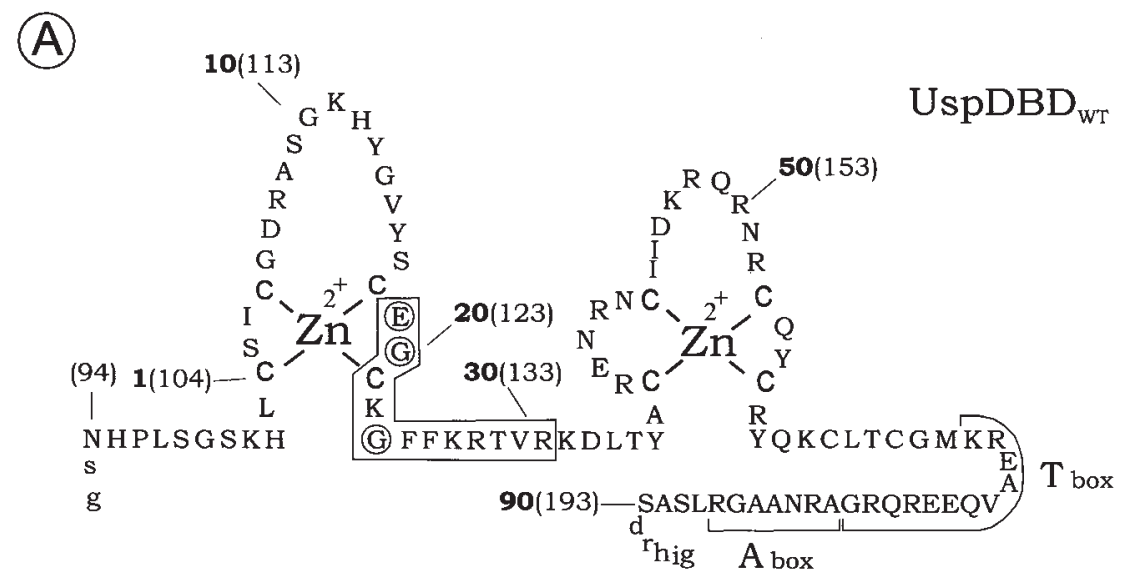

(B)

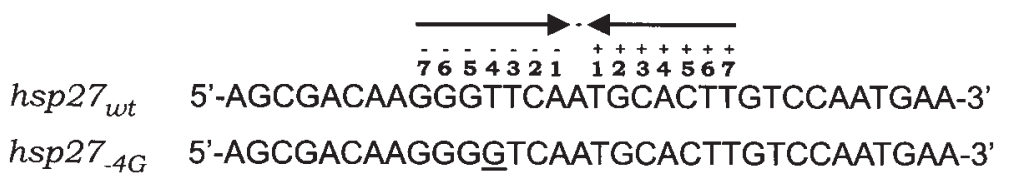

Figure 1. Sequences of the macromolecular components used in this study.

A. Amino-acid sequence of wild type UspDBD (UspDBD $\mathrm{WTT}_{\mathrm{WT}}$ ). The numbering in bold is relative to the first $\mathrm{Zn}$-coordinating cysteine of the $\mathrm{DBD}$, whereas numbers in parenthesis relate to the residue position relative to the N-terminus of full-length Drosophila melanogaster Usp (Oro et al., 1990). Residues in open circles correspond to the P-box amino acids; residues (19-31, except for C21) from the putative DNA recognition $\alpha$-helix that were substituted with alanine are boxed. Note that three of the mutant DBDs (F25A, F26A and V29A) could not be analyzed as they were unstable during purification (Grad et al., 2001). B. Sequences of the oligonucleotides used in electrophoretic mobility-shift assays. The sequences of the ds oligonucleotides are based on the sequence from the D. melanogaster hsp27 gene promoter (Riddihough \& Pelham, 1987; Ożyhar et al., 1991). hsp27 $7_{w t}$ contains a 15-bp semi-palindromic EcRE - marked with the arrows. $h s p 27_{-4 G}$ is $h s p 27_{w t}$ with base pair G/C in position -4; the exchanged nucleotide is underlined. The numbering convention in $h s p 27_{w t}$ was taken from previous study (Grad et al., 2001). For clearness of presentation only one strand of ds nucleotides is shown.

ity of UspDBD for a ds oligonucleotide containing hsp27 EcRE ( $h s p 27_{w t}$ ) (Fig. 2, compare lanes 1-3 and 4-6). However, one notable feature of many EcRE elements, including $h s p 27_{w t}$, is that a T/A base pair is present at their position -4 instead of a G/C (Riddihough \& Pelham, 1987; Antoniewski et al., 1993; Antoniewski et al., 1994; Lehmann \& Korge, 1995; Lehmann et al., 1997). To check if E19 exhibits a potential for binding to the -4 position, we substituted the T-4/A-4 base pair in $h s p 27_{w t}$ with G-4/C-4 to create hsp27-4G. This substitution significantly increased the DNA binding affinity of $\mathrm{UspDBD}_{\mathrm{WT}}$ in comparison with its affinity to hsp27 $7_{w t}$ (Fig. 2, compare lanes 1-3 and 7-9). Unexpectedly, the E19A mutant exhibited lower affinity for the $h s p 27_{-4 G}$ probe than to the wild-type sequence (Fig. 3 compare $3 \mathrm{~A}$ and 3B; Fig. 2, compare the ratio of $\mathrm{CI}_{\mathrm{U}}$ complexes intensity to the intensity of free DNA (F) in lanes 4, 5, 6 with lanes 10,11, 12). The above data may indicate that the interaction between the E19 residue and the G-4/C-4 base pair provides a substantial change in free energy, which overcomes some unfavorable interactions between UspDBD and the $h s p 27_{-4 G}$ element, which take place when E19 is absent, i.e. replaced by A. In contrast, removal of E19 did not reduce significantly the affinity of UspDBD for the $h s p 27_{w t}$ sequence (Fig. 2, compare lanes $1,2,3$ and 4, 5, 6). Together, these results suggest that in addition to E19 some other amino acids make the $h s p 27_{-4 G}$ se- 


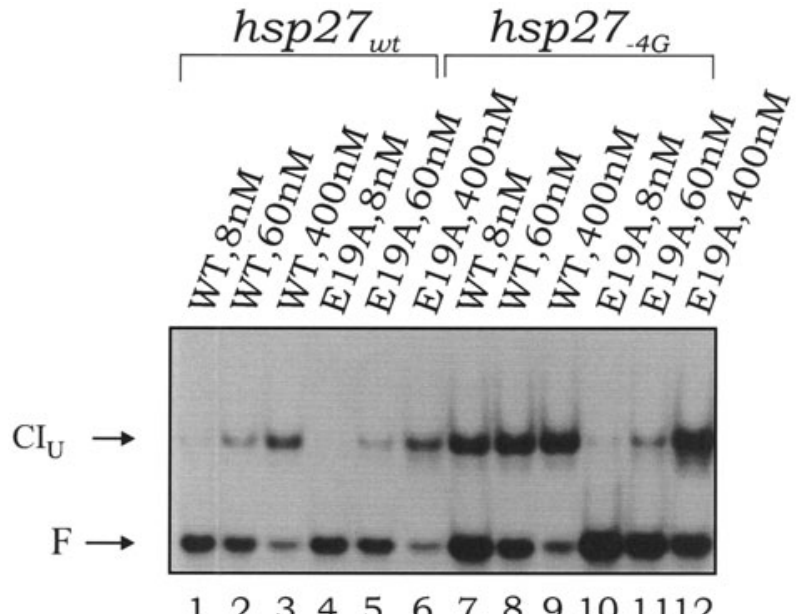

12233455667889101112

Figure 2. Comparison of $h s p 27_{w t}$ and hsp 27- $_{4 G}$ interaction with UspDBD and the E19A mutant.

Binding of wild type UspDBD (UspDBD $\left.{ }_{\mathrm{WT}}\right)$ (lanes 1-3 and 7-9) and E19A mutant (lanes $4-6$; $10-12)$ to the relevant element $-h s p 27_{w t}$ (lanes 1-6) and $h s p 27_{-4 G}$ (lanes 7-12) was studied. The proteins and their concentrations are indicated at the top. Monomeric complexes between UspDBD and DNA are indicated by $\mathrm{CI}_{\mathrm{U}} ; \mathrm{F}$, free probe. To estimate the relative binding activities compare the ratio of $\mathrm{CI}_{\mathrm{U}}$ complexes intensity to the intensity of free DNA in the respective lanes.

quence-specific contacts, which do not take place when $h s p 27_{w t}$ is used as a target sequence. To test this hypothesis we analyzed the interaction pattern of $h s p 27_{-4 G}$ with UspDBD mutants where individual amino acids of the putative recognition $\alpha$-helix were substituted with alanine (see Fig. 1A). The results presented in Fig. 4 indicate that substitution of the G20, K22, G23 T28 and K31 residues (P-box region amino acids) of UspDBD results in different magnitudes of effect on
DNA binding than it was observed for $h s p 27_{w t}$ (compare Fig. 4, lanes 3, 4, 5, 8, 10 and 13, 14, 15, 18 and 20, respectively). No clear differences were observed, however, when other amino-acid residues were mutated (compare Fig. 4, lanes 6, 7, 9, and 16, 17, 19, respectively). Thus, we conclude that the E19 residue of UspDBD seems to be not involved in any $h s p 27_{w t}$ sequence-discerning contacts. In contrast, when a G/C base pair is present at the -4 position of EcRE, E19 creates (possibly

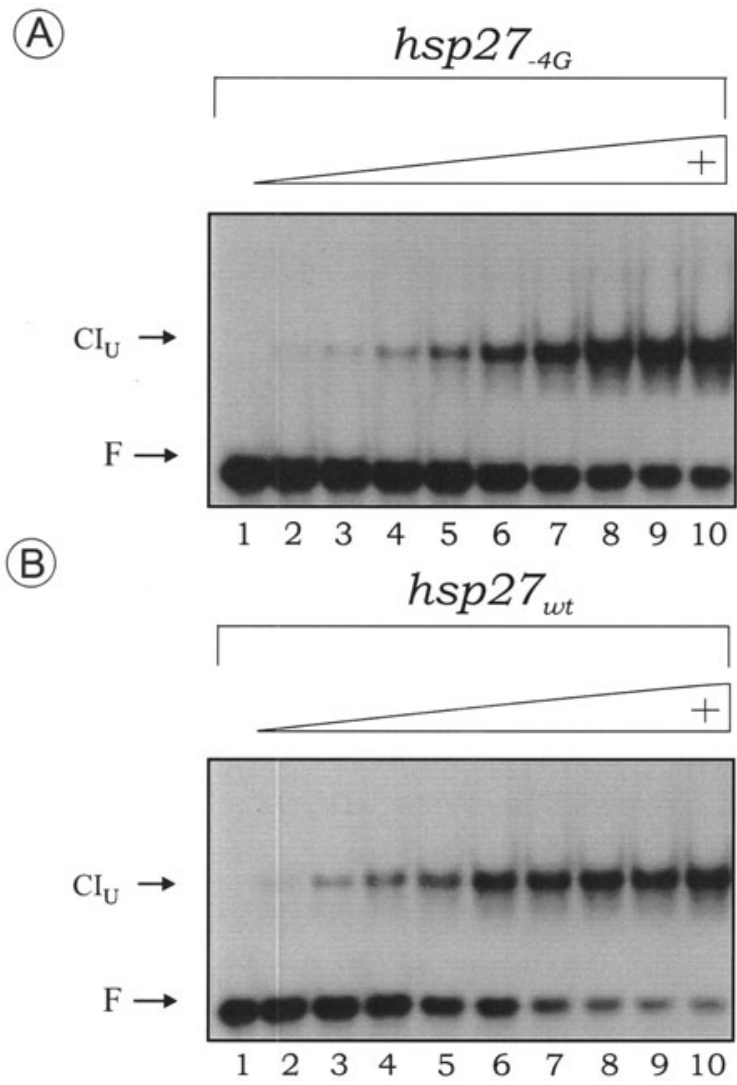

\section{$\mathrm{UspDBD}_{\mathrm{E} 19 \mathrm{~A}}$}

\section{Figure 3. Comparative DNA-binding $\mathrm{UspDBD}_{\mathrm{E} 19 \mathrm{~A}}$ of the E19A mutant of UspDBD to hsp27 $7_{-4 G}$ and to $h s p 27_{w t}$}

EMSAs were performed with the respective elements - $h s p 27_{-4 G}$ (A) or $h s p 27_{w t}$ (B) and increasing amounts of E19A UspDBD. Protein concentration (in nM) in lanes $1-10$ was $0,8,16,32,60,120$, 240, 400, 600, 800 . 
in co-operation with other P-box region amino-acid residues) protein-DNA contacts, which are distinct from those of $h s p 27_{w t}$ (summarized in Table 1). Notably, the same seems to be generally true for the $h s p 27_{-4 G}$ interaction with the UspDBD/EcRDBD heterodimer (Fig. 5). These observations suggest that substitution of the $h s p 27_{w t}$ T-4/A-4 base pair with G-4/C-4 would generate a target sequence with distinct functional properties and possibly with a new specificity. In the case of $20 \mathrm{E}$ receptor, most naturally occurring elements have the base pair T-4/A-4 (Riddihough \& Pelham, 1987; Antoniewski et al., 1993; 1994;
EcRDBD complex (Niedziela-Majka et al., 2000). Our preliminary results indicate that the $h s p 27_{-4 G}$ element binds EcRDBD with higher affinity than $h s p 27_{w t}$, but the presence of the G/C base pair at the -4 position appears to exclude the cooperative formation of the EcRDBD homodimeric complexes (data not shown). We therefore suggest that substitution of the T-4/A-4 base pair of $h s p 27_{w t}$ with $\mathrm{G} / \mathrm{C}$ might generate a more restrictive binding element, which possesses structural determinants that favor the binding of the UspDBD/EcRDBD heterodimer, but at the same time deter the binding of the EcRDBD

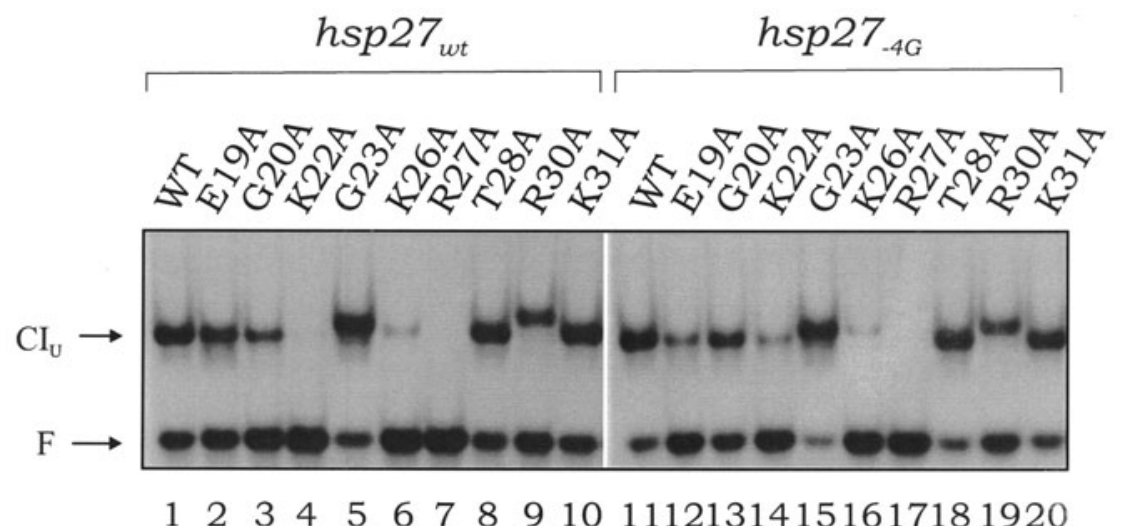

Figure 4. DNA-binding of UspDBD mutants to $h s p 27_{w t}$ and $h s p 27_{-4 G}$.

EMSAs were performed with $h s p 27_{w t}$ (lane 1-10) or $h s p 27_{-4 G}$ (lanes 11-20) and with $120 \mathrm{nM}$ of the UspDBDs (see Fig. 1A). Monomeric complexes between $\mathrm{UspDBD}_{\mathrm{N}} \mathrm{DNA}$ are indicated by $\mathrm{CI}_{\mathrm{U}}$; F, free probe. Note that, although for clarity the figure presents results only for one chosen amount of each DBD, for each protein and response element the experiment with a complete range of concentrations was performed, same as in Fig. 3 (not shown).

Lehmann \& Korge, 1995; Lehmann et al., 1997). The reason of this selection is unclear since binding site selection experiments (Vögtli et al., 1998) have shown that an oligonucleotide with the base pair G-4/C-4 binds the ecdysteroid receptor with the highest affinity. The basis for the T-4/A-4 selection might be associated with the ability to differentiate higher order complexes formation on EcREs with either a T/A or a G/C base pair in position -4. As was shown previously, $h s p 27_{w t}$ serves not only as a target for the UspDBD/ EcRDBD heterocomplex but contains all the structural information necessary for the synergistic formation of the homodimeric homodimer. In contrast, a T-4/A-4-containing target (i.e. $h s p 27_{w t}$ ) would interact in a clearly synergistic manner either with the UspDBD/ EcRDBD heterodimer or with the EcRDBD homodimer (Niedziela-Majka et al., 2000).

Although it is widely, but not universally, accepted that the functional $20 \mathrm{E}$ receptor is the heterodimer of Usp and EcR, we hypothesize that subtle nucleotide differences in the EcREs could provide structural basis for the discrimination of the Usp/EcR heterodimer vs. the EcR/EcR homodimer. The implications of this are that both dimers may contact some regulatory elements, for example $h s p 27_{w t}$, yet they may be elements that specifi- 
Table 1. Contacts of the E19 residue of known DBDs with bases of response elements.

Schematic representation of contacts between side chain of the E19 residue of DBDs with estrogen receptor-type P-box and REs based on the respective crystallographic data (A) and results presented in the study (B). In the left column the DBDs of respective receptors are denoted; in the case of dimeric structures the order of the proteins on the $\mathrm{RE}$ is kept. On the right the respective oligonucleotides used in the experiments with interactions with E19 marked are shown. W, water molecule. Underlined are half sites of REs, in italics the spacer between half sites. Note that additional contacts in the case of $h s p 27_{-4 G}$ are possible.

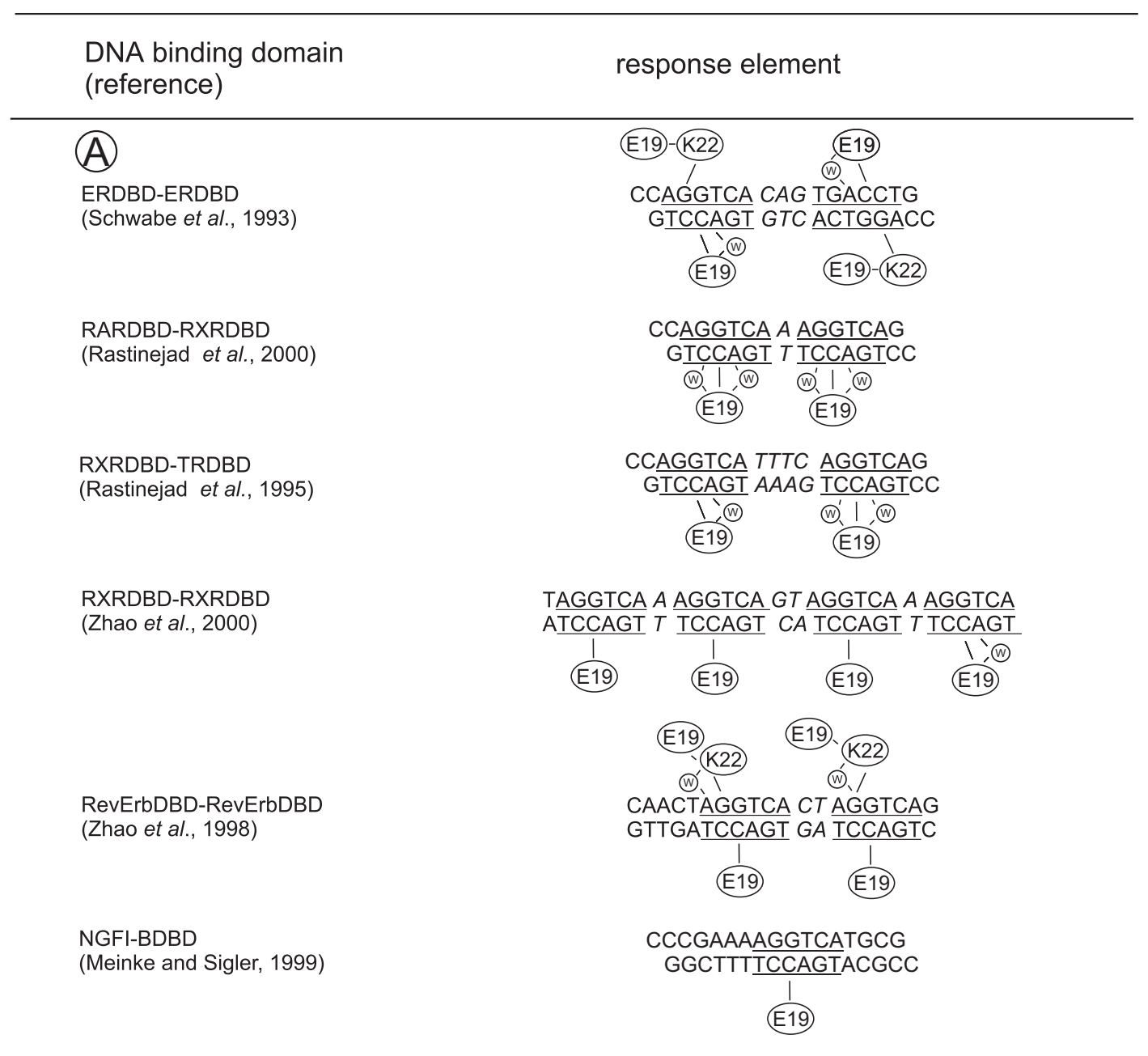

\begin{tabular}{|c|c|}
\hline (B) & \\
\hline $\begin{array}{l}\text { UspDBD } \\
\text { (this paper) }\end{array}$ & $\begin{array}{l}\text { AGCGACAAGGGTTCA } A \text { TGCACTTGTCCAATGAA } \\
\text { TCGCTGTTCCCAAGT } T \underline{\text { ACGTGAACAGGTTACTT }}\end{array}$ \\
\hline & $\begin{array}{l}\text { K } \\
\text { E19 }\end{array}\left(h s p 27_{w t}\right)$ \\
\hline $\begin{array}{l}\text { UspDBD } \\
\text { (this paper) }\end{array}$ & $\begin{array}{l}\text { AGCGACAAGGGGTCA } A \text { TGCACTTGTCCAATGAA } \\
\text { TCGCTGTTCCCCAGT } T \text { ACGTGAACAGGTTACTT }\end{array}$ \\
\hline & $\stackrel{1}{\text { E19 }} \quad\left(h s p 27_{-4 G}\right)$ \\
\hline
\end{tabular}

cally bind only the Usp/EcR heterodimer. This hypothesis requires further support by functional experiments, however, a compari- son of the natural EcREs clearly indicates that they contain either a T/A (Riddihough \& Pelham, 1987; Antoniewski et al., 1993; Lehmann 
(A)

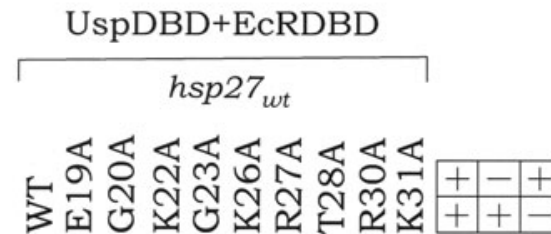

\section{EcRDBD \\ $\mathrm{UspDBD}_{\text {wт }}$}

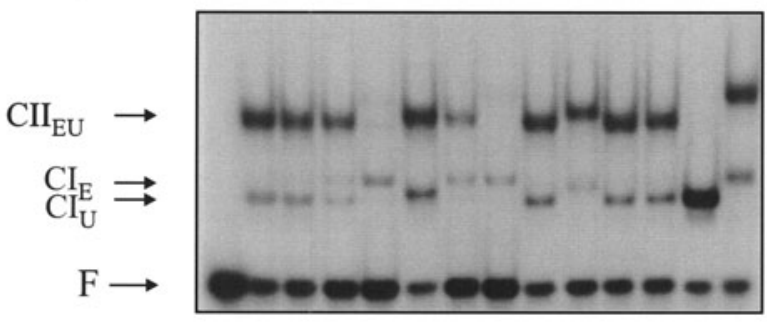

$\leftarrow \mathrm{CII}_{\mathrm{E}}$

122345567891011121314

(B)
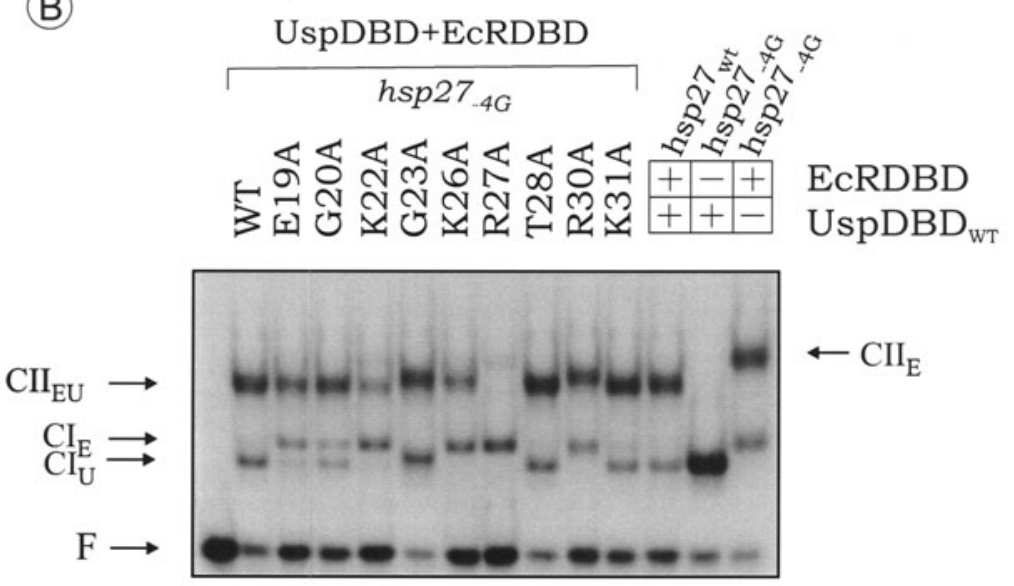

122345667891011121314

Figure 5. DNA-binding of UspDBD mutants together with EcRDBD to $h s p 27_{w t}$ and $h s p 27_{-4 G^{*}}$

EMSAs were performed with $h s p 27_{w t}$ (A, lanes 1-11) or $h s p 27_{-4 G}$ (B, lanes 1-11) and with $60 \mathrm{nM}$ of the purified UspDBDs indicated at the top and $60 \mathrm{nM}$ EcRDBD. The designations of the mutant UspDBD are the same as in Fig. 4. The complexes formed by one DBD molecule are indicated by CI and those originating from homo- or heterodimer by CII ; F, free probe. Lanes 12-14 in A controls: $h s p 27_{w t}$ with: 12-120 nM UspDBD ${ }_{\mathrm{WT}}$ and EcRDBD; 13-600 nM UspDBD ${ }_{\mathrm{WT}} ; 14-600 \mathrm{nM}$ EcRDBD. Lanes 12-14 in B controls: $12-h s p 27_{w t}$ with $120 \mathrm{nM} \mathrm{UspDBD} \mathrm{WT}_{\mathrm{WT}}$ and EcRDBD; $13-h s p 27_{-4 G}$ and $600 \mathrm{nM} \mathrm{UspDBD}{ }_{\mathrm{WT}} ; 14-h s p 27_{-4 G}$ and $600 \mathrm{nM}$ EcRDBD.

\& Korge, 1995; Lehmann et al., 1997) or a G/C (Cherbas et al., 1991; Antoniewski et al., 1995) base pair in the -4 position.

Authors thank Ms Mirosława Ostrowska for her excellent technical assistance.

\section{R E F E R E N C E S}

Antoniewski C, Laval M, Lepesant JA. (1993) Structural features critical to the activity of an ecdysone receptor binding site. Insect Biochem Mol Biol.; 23: 105-14.

Antoniewski C, Laval M, Dahan A, Lepesant JA. (1994) The ecdysone response enhancer of the Fbp 1 gene of Drosophila melanogaster is a direct target for the EcR/USP nuclear receptor. Mol Cell Biol.; 14: 4465-74.

Antoniewski C, O'Grady MS, Edmondson RG, Lassieur SM, Benes H. (1995) Characterization of an EcR/USP heterodimer target site that mediates ecdysone responsiveness of the 
Drosophila Lsp-2 gene. Mol Gen Genet.; 249: 545-56.

Barik S. (1995) Site-directed mutagenesis by double polymerase chain reaction. $\mathrm{Mol}$ Biotechnol.; 3: 1-7.

Cherbas L, Lee K, Cherbas P. (1991) Identification of ecdysone response elements by analysis of the Drosophila Eip28/29 gene. Genes Dev.; 5: 120-31.

Evans RM. (1988) The steroid and thyroid hormone receptor superfamily. Science.; 240: 889-95.

Freedman LP, Luisi BF, Korszun ZR, Basavappa R, Sigler PB, Yamamoto KR. (1988) The function and structure of the metal coordination sites within the glucocorticoid receptor DNA binding domain. Nature.; 334: 543-46.

Fried M, Crothers DM. (1981) Equilibria and kinetics of lac repressor-operator interactions by polyacrylamide gel electrophoresis. Nucleic Acids Res.; 9: 6505-25.

Gehring U. (1998) Steroid hormone receptors and heat shock proteins. Vitam Horm.; 54: 167-205.

Gill SC, von Hippel PH. (1989) Calculation of protein extinction coefficients from amino acid sequence data. Anal Biochem.; 182: $319-26$.

Grad I, Niedziela-Majka A, Kochman M, Ożyhar A. (2001) Analysis of Usp DNA-binding domain targeting reveals critical determinants of the ecdysone receptor complex interaction with the response element. Eur J Biochem.; 268: $3751-8$.

Green S, Kumar V, Theulaz I, Wahli W, Chambon P. (1988) The N-terminal DNA-binding 'zinc finger' of the oestrogen and glucocorticoid receptors determines target gene specificity. EMBO J.; 7: 3037-44.

Gronemeyer H, Laudet V. (1995) Transcription factors 3: nuclear receptors. Protein Profile.; 2: 1173-308.

Koelle MR, Talbot WS, Segraves WA, Bender MT, Cherbas P, Hogness DS. (1991) The Drosophila EcR gene encodes an ecdysone re- ceptor, a new member of the steroid receptor superfamily. Cell.; 67: 59-77.

Kozlova T, Thummel CS. (2000) Steroid regulation of postembryonic development and reproduction in Drosophila. Trends Endocrin Metab.; 11: 276-80.

Lehmann M, Korge G. (1995) Ecdysone regulation of the Drosophila Sgs-4 gene is mediated by the synergistic action of ecdysone receptor and SEBP 3. EMBO J.; 14: 716-26.

Lehmann M, Wattler F, Korge G. (1997) Two new regulatory elements controlling the Drosophila Sgs-3 gene are potential ecdysone receptor and fork head binding sites. Mech Dev.; 62: 15-27.

Mader S, Kumar V, de Verneuil H, Chambon P. (1989) Three amino acids of the oestrogen receptor are essential to its ability to distinguish an oestrogen from a glucocorticoid-responsive element. Nature.; 338: 271-4.

Meinke G, Sigler PB. (1999) DNA-binding mechanism of the monomeric orphan nuclear receptor NGFI-B. Nat Struct Biol.; 6: 471-7.

Nelson CC, Hendy SC, Shukin RJ, Cheng H, Bruchovsky N, Koop BF, Rennie PS. (1999) Determinants of DNA sequence specificity of the androgen, progesterone, and glucocorticoid receptors: evidence for differential steroid receptor response elements. Mol Endocrinol.; 13: 2090-107.

Niedziela-Majka A, Rymarczyk G, Kochman M, Ożyhar A. (1998) Pure, bacterially expressed DNA-binding domains of the functional ecdysteroid receptor capable of interacting synergistically with the $h s p 27$ 20-hydroxyecdysone response element. GST-Induced dimerization of DNA-binding domains alters characteristics of their interaction with DNA. Protein Expr Purif.; 14: 208-20.

Niedziela-Majka A, Kochman M, Ożyhar A. (2000) Polarity of the ecdysone receptor complex interaction with the palindromic response element from the $h s p 27$ gene promoter. Eur J Biochem.; 267: 507-19. 
Oro AE, McKeown M, Evans RM. (1990) Relationship between the product of the Drosophila ultraspiracle locus and the vertebrate retinoid X receptor. Nature.; 347: 298-301.

Ożyhar A, Strangmann-Diekmann M, Kiltz HH, Pongs O. (1991) Characterization of a specific ecdysteroid receptor-DNA complex reveals common properties for invertebrate and vertebrate hormone-receptor/DNA interactions. Eur J Biochem.; 200: 329-35.

Rastinejad F, Perlmann T, Evans RM, Sigler PB. (1995) Structural determinants of nuclear receptor assembly on DNA direct repeats. $\mathrm{Na}$ ture.; 375: 203-11.

Rastinejad F, Wagner T, Zhao Q, Khorasanizadeh S. (2000) Structure of the RXR-RAR DNA-binding complex on the retinoic acid response element DR1. EMBO J.; 19: 1045-54.

Renaud JP, Moras D. (2000) Structural studies on nuclear receptors. Cell Mol Life Sci.; 57: 1748-69.

Riddihough G, Pelham HRB. (1987) An ecdysone response element in the Drosophila hsp27 promoter. EMBO J.; 6: 3729-34.

Schwabe JW, Chapman L, Finch JT, Rhodes D. (1993) The crystal structure of the estrogen receptor DNA-binding domain bound to DNA: how receptors discriminate between their response elements. Cell.; 75: 567-78.

Sluder AE, Maina CV. (2001) Nuclear receptors in nematodes: themes and variations. Trends Genet.; 17: 206-13.
Smith DB, Johnson KS. (1988) Single-step purification of polypeptides expressed in Escherichia coli as fusions with glutathione S-transferase. Gene.; 67: 31-40.

Studier FW. (1991) Use of bacteriophage T7 lysozyme to improve an inducible T7 expression system. J Mol Biol.; 219: 37-44.

Thomas HE, Stunnenberg HG, Stewart AF. (1993) Heterodimerization of the Drosophila ecdysone receptor with retinoid $\mathrm{X}$ receptor and ultraspiracle. Nature.; 362: 471-5.

Torchia J, Glass C, Rosenfeld MG. (1998) Co-activators and co-repressors in the integration of transcriptional responses. Curr Opin Cell Biol.; 10: 373-83.

Umesono K, Evans RM. (1989) Determinants of target gene specificity for steroid/thyroid hormone receptors. Cell.; 57: 1139-46.

Vögtli M, Elke C, Imhof MO, Lezzi M. (1998) High level transactivation by the ecdysone receptor complex at the core recognition motif. Nucleic Acids Res.; 26: 2407-14.

Yao TP, Segraves WA, Oro AE, McKeown M, Evans RM. (1992) Drosophila ultraspiracle modulates ecdysone receptor function via heterodimer formation. Cell.; 71: 63-72.

Zhao Q, Khorasanizadeh S, Miyoshi Y, Lazar MA, Rastinejad F. (1998) Structural elements of an orphan nuclear receptor-DNA complex. Mol Cell.; 1: 849-61.

Zhao Q, Chasse SA, Devarakonda S, Sierk ML, Ahvazi B, Rastinejad F. (2000) Structural basis of RXR-DNA interactions. $J$ Mol Biol.; 296: 509-20. 